\title{
Article \\ A Generalized Two-Dimensional Index to Measure the Degree of Deviation from Double Symmetry in Square Contingency Tables
}

\author{
Shuji Ando ${ }^{1, *\left(\mathbb{D}, \text { Hikaru Hoshi }^{2}, \text { Aki Ishii }^{2} \text { and Sadao Tomizawa }\right.}{ }^{2}$ \\ 1 Department of Information and Computer Technology, Faculty of Engineering, Tokyo University of Science, \\ Tokyo 125-8585, Japan \\ 2 Department of Information Sciences, Faculty of Science and Technology, Tokyo University of Science, \\ Chiba 278-8510, Japan; 6318528@alumni.tus.ac.jp (H.H.); a.ishii@rs.tus.ac.jp (A.I.); \\ tomizawa@is.noda.tus.ac.jp (S.T.) \\ * Correspondence: shuji.ando@rs.tus.ac.jp
}

check for updates

Citation: Ando, S.; Hoshi, H.; Ishii,

A.; Tomizawa, S. A Generalized

Two-Dimensional Index to Measure the Degree of Deviation from Double Symmetry in Square Contingency

Tables. Symmetry 2021, 13, 2067.

https://doi.org/10.3390/sym13112067

Academic Editor: Alice Miller

Received: 28 September 2021

Accepted: 27 October 2021

Published: 2 November 2021

Publisher's Note: MDPI stays neutral with regard to jurisdictional claims in published maps and institutional affiliations.

Copyright: (c) 2021 by the authors. Licensee MDPI, Basel, Switzerland. This article is an open access article distributed under the terms and conditions of the Creative Commons Attribution (CC BY) license (https:// creativecommons.org/licenses/by/ $4.0 /)$.

\begin{abstract}
The double symmetry model satisfies both the symmetry and point symmetry models simultaneously. To measure the degree of deviation from the double symmetry model, a twodimensional index that can concurrently measure the degree of deviation from symmetry and point symmetry is considered. This two-dimensional index is constructed by combining two existing indexes. Although the existing indexes are constructed using power divergence, the existing twodimensional index that can concurrently measure both symmetries is constructed using only KullbackLeibler information, which is a special case of power divergence. Previous studies note the importance of using several indexes of divergence to compare the degrees of deviation from a model for several square contingency tables. This study, therefore, proposes a two-dimensional index based on power divergence in order to measure deviation from double symmetry for square contingency tables. Numerical examples show the utility of the proposed two-dimensional index using two datasets.
\end{abstract}

Keywords: confidence region; measure; point symmetry; power divergence; symmetry

\section{Introduction}

Consider an $r \times r$ square contingency table that has the same row and column classifications with nominal categories. Let $\pi_{i j}$ denote the probability that an observation will fall in the $i$ th row and $j$ th column of the table $(i=1, \ldots, r ; j=1, \ldots, r)$.

The symmetry (S) model proposed by Bowker [1] is defined by

$$
\pi_{i j}=\pi_{j i} \quad \text { for } \quad i \neq j \text {. }
$$

This $\mathrm{S}$ model is the most commonly used model for analyzing square contingency tables [2-4].

The point symmetry (PS) model proposed by Wall and Lienert [5] is defined by

$$
\pi_{i j}=\pi_{i^{*} j^{*}} \quad \text { for } \quad i, j=1, \ldots, r,
$$

where $i^{*}=r+1-i$ and $j^{*}=r+1-j$. This PS model assumes the point of symmetry as a center of the square contingency table.

The double symmetry (DS) model proposed by Tomizawa [6] is defined by

$$
\pi_{i j}=\pi_{j i}=\pi_{i^{*} j^{*}}=\pi_{j^{*} i^{*}} \text { for } \quad i, j=1, \ldots, r .
$$

This DS model indicates that both the $S$ and PS model hold.

When a model does not hold, we may be interested in measuring the degree of deviation from the model. For square contingency tables with nominal categories, Tomizawa 
et al. [7] proposed an index $\Phi_{S}^{(\lambda)}$ that represents the degree of deviation from the $S$ model, Tomizawa et al. [8] proposed an index $\Phi_{P S}^{(\lambda)}$ that represents the degree of deviation from the PS model, and Yamamoto et al. [9] proposed an index $\Phi_{D S}^{(\lambda)}$ that represents the degree of deviation from the DS model.

This study focuses on the index that represents the degree of deviation from the DS model. Although the DS model satisfies both the S and PS models simultaneously, the above index $\Phi_{D S}^{(\lambda)}$ cannot concurrently measure the degree of deviation from $S$ and PS. To address this gap, Ando et al. [10] proposed a two-dimensional index that can concurrently measure those. This two-dimensional index was constructed by combining existing indexes $\Phi_{S}^{(\lambda)}$ and $\Phi_{P S}^{(\lambda)}$. Ando et al. [10] points out that it is necessary to construct as a two-dimensional index rather than a univariate index because existing indexes $\Phi_{S}^{(\lambda)}$ and $\Phi_{P S}^{(\lambda)}$ are not independent. Ando et al. [10] considered three datasets: (1) the degree of deviation from the $S$ model is large but the degree of deviation from the PS model is small, (2) the degree of deviation from the $S$ model is small but the degree of deviation from the PS model is large, and (3) both the degree of deviation from the $S$ model and the PS model are large. By using these datasets which have a different structure with respect to the deviation from the DS model, Ando et al. [10] showed that the all values of the index $\Phi_{D S}^{(\lambda)}$ applied to these datasets are the same, whereas all the values of the two-dimensional index are different. Thus, this two-dimensional index gives more detailed results than the index $\Phi_{D S}^{(\lambda)}$.

On the other hand, existing indexes $\Phi_{S}^{(\lambda)}, \Phi_{P S}^{(\lambda)}$ and $\Phi_{D S}^{(\lambda)}$ are constructed using power divergence, while the two-dimensional index is constructed using only Kullback-Leibler information, which is a special case of power divergence. Moreover, the power divergence includes several divergences, for example, the power divergence with $\lambda=-0.5$ is equivalent to the Freeman-Tukey type divergence, the power divergence with $\lambda=1$ is equivalent to the Pearson chi-squared type divergence. For details on power divergence, see Cressie and Read [11], Read and Cressie [12]. Previous studies (e.g., [7,8]) pointed out that it is important to use several indexes of divergence to accurately measure the degree of deviation from a model. This study proposes a two-dimensional index that is constructed by combining existing indexes $\Phi_{S}^{(\lambda)}$ and $\Phi_{P S}^{(\lambda)}$ based on power divergence.

The rest of this paper is organized as follows. In Section 2, we propose a generalized two-dimensional index for measuring the degree of deviation from DS. In Section 3, we develop an approximate confidence region for the proposed two-dimensional index. We then use numerical examples to show the utility of the proposed two-dimensional index in Section 4. We also present results obtained by applying the proposed two-dimensional index to real data. We close with concluding remarks in Section 5.

\section{Two-Dimensional Index to Measure Deviation from DS}

We propose a generalized two-dimensional index for measuring deviation from DS in square contingency tables. The proposed two-dimensional index can concurrently measure the degree of deviation from S and PS. The proposed two-dimensional index is based on power divergence.

Assume that $\pi_{i j}+\pi_{j i}>0$ for all $i \neq j$, and $\pi_{i j}+\pi_{i^{*} j^{*}}>0$ for all $(i, j) \in E$, where

$$
E= \begin{cases}\{(i, j) \mid i, j=1, \ldots, r ;(i, j) \neq((r+1) / 2,(r+1) / 2)\} & (r \text { is odd }) \\ \{(i, j) \mid i, j=1, \ldots, r\} & (r \text { is even }) .\end{cases}
$$

In order to measure the degree of deviation from DS, we consider the following two-dimensional index:

$$
\Psi^{(\lambda)}=\left(\begin{array}{c}
\Phi_{S}^{(\lambda)} \\
\Phi_{P S}^{(\lambda)}
\end{array}\right) \quad(\lambda>-1)
$$


where indexes $\Phi_{S}^{(\lambda)}$ and $\Phi_{P S}^{(\lambda)}$ are those considered by Tomizawa et al. [7] and Tomizawa et al. [8], respectively (see the Appendixes A and B for the details of these indexes). Note that the $\lambda$ is a real value and is chosen by the user. We recommend choosing the $\lambda$ (e.g., $-0.5,0,1)$ corresponding to the famous divergence. When $\lambda=0$, the proposed two-dimensional index is equivalent to the index by Ando et al. [10]. Thus, $\Psi^{(\lambda)}$ is a generalization of the index by Ando et al. [10]. The two-dimensional index $\Psi^{(\lambda)}$ has the following characteristics: (i) $\Psi^{(\lambda)}=(0,0)^{\prime}$ if and only if the DS model holds; (ii) $\Psi^{(\lambda)}=(1,1)^{\prime}$ if and only if the degree of deviation from DS is maximum, in the sense that $\pi_{i j}=\pi_{j^{*} i^{*}}=0$ (then $\pi_{j i}>0$ and $\pi_{i^{*} j^{*}}>0$ ) or $\pi_{j i}=\pi_{i^{*} j^{*}}=0$ (then $\pi_{i j}>0$ and $\pi_{j^{*} i^{*}}>0$ ) for all $i \neq j$, and either $\pi_{i i}=0$ or $\pi_{i^{*} i^{*}}=0$ for $i=1, \ldots, r / 2$ (when $r$ is even) or $i=1, \ldots,(r-1) / 2$ (when $r$ is odd); (iii) $\Psi^{(\lambda)}=(1, * *)^{\prime}$ if and only if the degree of deviation from $\mathrm{S}$ is maximum and the degree of deviation from PS is not maximum, in the sense that $\pi_{i j}=0$ (then $\pi_{j i}>0$ ) for all $i \neq j$; and (iv) $\Psi^{(\lambda)}=(* *, 1)^{\prime}$ if and only if the degree of deviation from PS is maximum and the degree of deviation from $\mathrm{S}$ is not maximum, in the sense that $\pi_{i j}=0\left(\right.$ then $\pi_{i^{*} j^{*}}>0$ ) for all $(i, j) \in E$.

\section{Approximate Confidence Region for the Proposed Two-Dimensional Index}

Let

$$
\begin{gathered}
\boldsymbol{n}=\left(n_{11}, n_{12}, \ldots, n_{1 r}, n_{21}, n_{22}, \ldots, n_{2 r}, \ldots, n_{r 1}, n_{r 2}, \ldots, n_{r r}\right)^{\prime}, \\
\boldsymbol{\pi}=\left(\pi_{11}, \pi_{12}, \ldots, \pi_{1 r}, \pi_{21}, \pi_{22}, \ldots, \pi_{2 r}, \ldots, \pi_{r 1}, \pi_{r 2}, \ldots, \pi_{r r}\right)^{\prime} .
\end{gathered}
$$

Assume that $n$ has a multinomial distribution with sample size $N$ and probability vector $\pi$. The $\sqrt{N}(\boldsymbol{p}-\boldsymbol{\pi})$ has an asymptotically Gaussian distribution with mean zero and covariance matrix $\boldsymbol{D}(\boldsymbol{\pi})-\pi \pi^{\prime}$, where $\boldsymbol{p}=n / N$ and $\boldsymbol{D}(\boldsymbol{\pi})$ is a diagonal matrix with the elements of $\pi$ on the main diagonal (see, e.g., Agresti [13]). We estimate $\Psi^{(\lambda)}$ by $\hat{\mathbf{\Psi}}^{(\lambda)}=\left(\hat{\Phi}_{S}^{(\lambda)}, \hat{\Phi}_{P S}^{(\lambda)}\right)^{\prime}$, where $\hat{\Phi}_{S}^{(\lambda)}$ and $\hat{\Phi}_{P S}^{(\lambda)}$ are given by $\Phi_{S}^{(\lambda)}$ and $\Phi_{P S}^{(\lambda)}$ with $\pi_{i j}$ replaced by $p_{i j}$, respectively. Using the delta method (see Agresti [13]), $\sqrt{N}\left(\hat{\mathbf{\Psi}}^{(\lambda)}-\mathbf{\Psi}^{(\lambda)}\right)$ has an asymptotically bivariate Gaussian distribution with mean zero and covariance matrix

$$
\begin{aligned}
\boldsymbol{\Sigma}^{(\lambda)} & =\left(\frac{\partial \Psi^{(\lambda)}}{\partial \pi^{\prime}}\right)\left(\boldsymbol{D}(\boldsymbol{\pi})-\boldsymbol{\pi} \boldsymbol{\pi}^{\prime}\right)\left(\frac{\partial \Psi^{(\lambda)}}{\partial \boldsymbol{\pi}^{\prime}}\right)^{\prime} \\
& =\left(\begin{array}{cc}
\sigma_{11}^{(\lambda)} & \sigma_{12}^{(\lambda)} \\
\sigma_{21}^{(\lambda)} & \sigma_{22}^{(\lambda)}
\end{array}\right)
\end{aligned}
$$

with $\sigma_{12}^{(\lambda)}=\sigma_{21}^{(\lambda)}$. Let

$$
\delta=\sum_{i \neq j} \pi_{i j}, \quad \Delta=\sum_{(i, j) \in E} \pi_{i j} .
$$

The elements $\sigma_{11}^{(\lambda)}, \sigma_{12}^{(\lambda)}$, and $\sigma_{22}^{(\lambda)}$ are expressed as follows:

$$
\begin{aligned}
\sigma_{11}^{(\lambda)} & =\left(\frac{\partial \Phi_{S}^{(\lambda)}}{\partial \pi^{\prime}}\right)\left(\boldsymbol{D}(\boldsymbol{\pi})-\pi \pi^{\prime}\right)\left(\frac{\partial \Phi_{S}^{(\lambda)}}{\partial \pi^{\prime}}\right)^{\prime} \\
& =\frac{1}{\delta^{2}}\left[\sum_{i \neq j} \pi_{i j}\left(\Omega_{i j}^{(\lambda)}\right)^{2}-\delta\left(\Phi_{S}^{(\lambda)}\right)^{2}\right]^{\prime} \\
\sigma_{12}^{(\lambda)} & =\left(\frac{\partial \Phi_{S}^{(\lambda)}}{\partial \pi^{\prime}}\right)\left(\boldsymbol{D}(\pi)-\pi \pi^{\prime}\right)\left(\frac{\partial \Phi_{P S}^{(\lambda)}}{\partial \pi^{\prime}}\right)^{\prime} \\
& =\frac{1}{\delta \Delta}\left[\sum_{i \neq j} \sum_{i j}\left(\Omega_{i j}^{(\lambda)}-\Phi_{S}^{(\lambda)}\right)\left(W_{i j}^{(\lambda)}-\Phi_{P S}^{(\lambda)}\right)\right],
\end{aligned}
$$




$$
\begin{aligned}
\sigma_{22}^{(\lambda)} & =\left(\frac{\partial \Phi_{P S}^{(\lambda)}}{\partial \pi^{\prime}}\right)\left(\boldsymbol{D}(\boldsymbol{\pi})-\pi \boldsymbol{\pi}^{\prime}\right)\left(\frac{\partial \Phi_{P S}^{(\lambda)}}{\partial \pi^{\prime}}\right)^{\prime} \\
& =\frac{1}{\Delta^{2}}\left[\sum_{i, j} \sum_{i \in E} \pi_{i j}\left(W_{i j}^{(\lambda)}\right)^{2}-\Delta\left(\Phi_{P S}^{(\lambda)}\right)^{2}\right],
\end{aligned}
$$

where for $\lambda>-1$

$$
\begin{aligned}
& \Omega_{i j}^{(\lambda)}= \begin{cases}\frac{1}{\log 2} \log \left(2 a_{i j}^{c}\right) & (\lambda=0), \\
\frac{1}{2^{\lambda}-1}\left[\left(2 a_{i j}^{c}\right)^{\lambda}-1+\lambda a_{j i}^{c}\left\{\left(2 a_{i j}^{c}\right)^{\lambda}-\left(2 a_{j i}^{c}\right)^{\lambda}\right\}\right] & (\lambda \neq 0),\end{cases} \\
& W_{i j}^{(\lambda)}= \begin{cases}\frac{1}{\log 2} \log \left(2 c_{i j}^{c}\right) & (\lambda=0), \\
\frac{1}{2^{\lambda}-1}\left[\left(2 c_{i j}^{c}\right)^{\lambda}-1+\lambda c_{i^{*} j^{*}}^{c}\left\{\left(2 c_{i j}^{c}\right)^{\lambda}-\left(2 c_{i^{*} j^{*}}^{c}\right)^{\lambda}\right\}\right] & (\lambda \neq 0),\end{cases}
\end{aligned}
$$

with

$$
a_{i j}^{c}=\frac{\pi_{i j}}{\pi_{i j}+\pi_{j i}}, \quad c_{i j}^{c}=\frac{\pi_{i j}}{\pi_{i j}+\pi_{i^{*} j^{*}}} .
$$

Note that the asymptotic variances $\sigma_{11}^{(\lambda)}$ and $\sigma_{22}^{(\lambda)}$ of $\Phi_{S}^{(\lambda)}$ and $\Phi_{P S}^{(\lambda)}$, respectively, have been given by Tomizawa et al. [7] and Tomizawa et al. [8], however, the asymptotic covariance $\sigma_{12}^{(\lambda)}$ of $\Phi_{S}^{(\lambda)}$ and $\Phi_{P S}^{(\lambda)}$ is first derived in this study. An approximate bivariate $100(1-\alpha) \%$ confidence region for the index $\Psi^{(\lambda)}$ is given by

$$
N\left(\hat{\Psi}^{(\lambda)}-\Psi^{(\lambda)}\right)^{\prime} \widehat{\boldsymbol{\Sigma}}^{-1}{ }^{-1}\left(\hat{\mathbf{\Psi}}^{(\lambda)}-\mathbf{\Psi}^{(\lambda)}\right) \leq \chi_{(1-\alpha ; 2)}^{2}
$$

where $\chi_{(1-\alpha ; 2)}^{2}$ is the upper $1-\alpha$ percentile of the central chi-square distribution with two degrees of freedom and $\widehat{\boldsymbol{\Sigma}^{(\lambda)}}$ is given by $\boldsymbol{\Sigma}^{(\lambda)}$ with $\pi_{i j}$ replaced by $p_{i j}$.

\section{Examples}

\subsection{Utility of the Proposed Two-Dimensional Index}

In this section, we demonstrate the usefulness employing several divergences to

\begin{tabular}{|c|c|c|c|c|c|c|c|}
\hline \multicolumn{4}{|l|}{ (a) } & \multicolumn{4}{|l|}{ (b) } \\
\hline 137 & 71 & 948 & 986 & 801 & 247 & 132 & 104 \\
\hline 291 & 605 & 400 & 997 & 964 & 973 & 56 & 406 \\
\hline 1 & 450 & 268 & 361 & 85 & 952 & 333 & 393 \\
\hline 22 & 645 & 639 & 124 & 809 & 697 & 625 & 727 \\
\hline
\end{tabular}
compare the degrees of deviation from DS in several datasets. We consider the two artificial datasets in Table 1. We compare the degrees of deviation from DS for Table 1a,b using the confidence region for $\Psi^{(\lambda)}$. Table 2 gives the estimated values of $\Psi^{(\lambda)}$ and $\boldsymbol{\Sigma}^{(\lambda)}$ for Table $1 \mathrm{a}, \mathrm{b}$.

Table 1. Two artificial datasets. 
Table 2. Estimated indexes $\hat{\Phi}_{S}^{(\lambda)}$ and $\hat{\Phi}_{P S}^{(\lambda)}$ and estimated covariance matrix of $\Psi^{(\lambda)}$ applied to the data in Table 1a,b.

(a) For Table 1a

\begin{tabular}{llllll}
\hline & \multicolumn{3}{c}{ Index } & \multicolumn{3}{c}{ Covariate Matrix } \\
\cline { 2 - 6 }$\lambda$ & $\hat{\mathbf{\Phi}}_{S}^{(\lambda)}$ & $\hat{\boldsymbol{\Phi}}_{P S}^{(\lambda)}$ & $\hat{\sigma}_{\mathbf{1 1}}^{(\lambda)}$ & $\hat{\sigma}_{\mathbf{1 2}}^{(\lambda)}$ & $\hat{\sigma}_{\mathbf{2 2}}^{(\lambda)}$ \\
\hline 0 & 0.346 & 0.341 & 0.471 & 0.278 & 0.417 \\
1 & 0.371 & 0.370 & 0.472 & 0.267 & 0.416 \\
\hline
\end{tabular}

(b) For Table $1 b$

\begin{tabular}{cccccc}
\hline & \multicolumn{3}{c}{ Index } & \multicolumn{3}{c}{ Covariate Matrix } \\
\cline { 2 - 6 }$\lambda$ & $\hat{\boldsymbol{\Phi}}_{S}^{(\lambda)}$ & $\hat{\boldsymbol{\Phi}}_{\boldsymbol{P S}}^{(\lambda)}$ & $\hat{\sigma}_{\mathbf{1 1}}^{(\lambda)}$ & $\hat{\sigma}_{\mathbf{1 2}}^{(\lambda)}$ & $\hat{\sigma}_{\mathbf{2 2}}^{(\lambda)}$ \\
\hline 0 & 0.287 & 0.259 & 0.853 & 0.488 & 0.538 \\
1 & 0.348 & 0.320 & 1.006 & 0.557 & 0.682 \\
\hline
\end{tabular}

From Figure 1, we see that the confidence regions for $\Psi^{(\lambda)}$ do not overlap for the data in Table 1a,b. We can conclude that Table $1 \mathrm{a}, \mathrm{b}$ has a different structure in the degree of deviation from DS. That is, Table $1 \mathrm{a}, \mathrm{b}$ has a different structure with regard to the degree of deviation from S or PS. From Figure 1, when $\lambda=0$, we can conclude that the degree of deviation from DS for Table 1a is greater than that for Table $1 \mathrm{~b}$, but when $\lambda=1$, we cannot conclude this. We should, therefore, examine the value of the two-dimensional index using several $\lambda$ to compare the degrees of deviation from DS for several datasets.

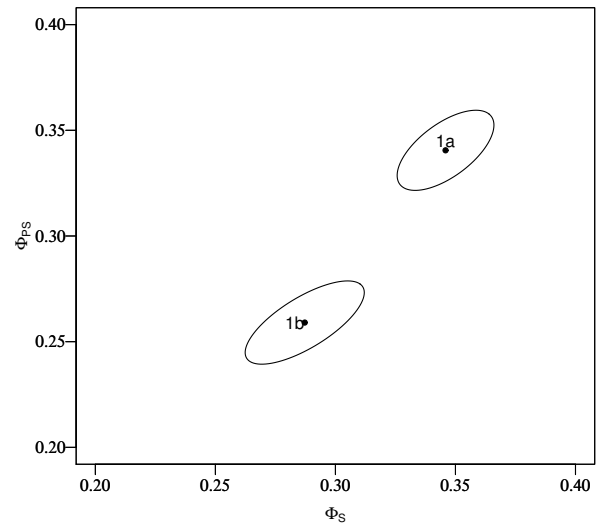

(a) $\lambda=0$

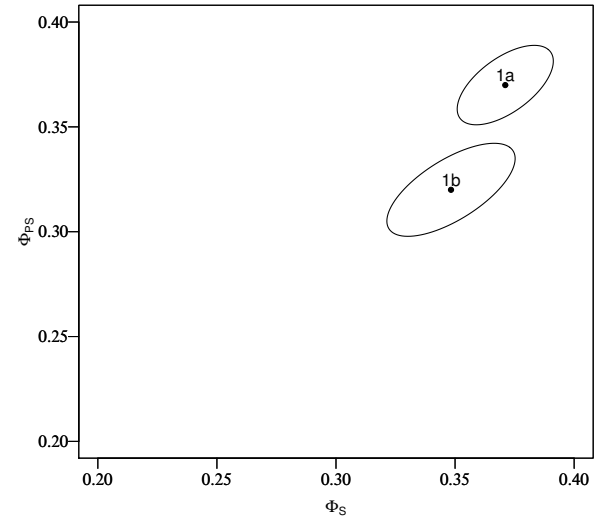

(b) $\lambda=1$

Figure 1. Aproximate $95 \%$ confidence regions for $\Psi^{(\lambda)}$ applied to the data in Table 1a,b.

\subsection{Example with Real Data}

Consider the data in Table 3, which are taken from Anderson [14].

We are interested in the DS model for these data. We define, for example, the probability that the forecast and actual figures are "No change" and "Higher", respectively, as $\pi_{N, H}$, and the probability that they are "Lower" and "No change", respectively, by $\pi_{L, N}$. For Table 3, we are interested in whether the forecast accuracy changes depending on the category. When the forecast accuracy does not depend on these categories, the following holds: (1) the probabilities that the categories of the forecast and the actual are the same and are equal to one another $\left(\pi_{H, H}=\pi_{L, L}\right)$; (2) the probabilities that the difference between the categories of the forecast and the actual is one are also equal $\left(\pi_{N, H}=\pi_{H, N}=\pi_{N, L}=\pi_{L, N}\right)$; and (3) the probabilities that the difference between the categories of the forecast and the actual is two are also equal $\left(\pi_{H, L}=\pi_{L, H}\right)$. The above probability structure indicates the DS model. Moreover, we are interested in whether the degree to which the forecast accuracy depends on the categories is greater for prices than for production, or vice versa. Table 4 shows the value of $\hat{\Phi}_{S}^{(\lambda)}$ and $\hat{\Phi}_{P S}^{(\lambda)}$. We shall compare the degrees of deviation from DS for 
Table 3a,b using the confidence region for $\Psi^{(\lambda)}$. The estimates of $\boldsymbol{\Sigma}^{(\lambda)}$, applied to the data in Table 3a,b, are shown in Table 4.

Table 3. The two tables below show the three-year production and price forecasts, given by experts in July 1956, and the actual production and price figures from May 1959 for a sample of about 4000 Danish factories; from Andersen [14].

\begin{tabular}{ccccc}
\hline (a) For prices & \multicolumn{4}{c}{ Actual } \\
\hline & \multicolumn{5}{c}{ Lower } & Total \\
\cline { 2 - 5 } Forecast & Higher & No Change & 6 & 384 \\
Higher & 209 & 169 & 184 & 3447 \\
No change & 190 & 3073 & 81 & 146 \\
Lower & 3 & 62 & 271 & 3977 \\
Total & 402 & 3304 & & \\
\hline
\end{tabular}

(b) For production

\begin{tabular}{ccccc}
\hline & \multicolumn{4}{c}{ Actual } \\
\cline { 2 - 5 } Forecast & Higher & No Change & Lower & Total \\
\hline Higher & 532 & 394 & 69 & 995 \\
No change & 447 & 1727 & 334 & 2508 \\
Lower & 39 & 230 & 231 & 500 \\
Total & 1018 & 2351 & 634 & 4003 \\
\hline
\end{tabular}

Table 4. Estimated indexes $\hat{\Phi}_{S}^{(\lambda)}$ and $\hat{\Phi}_{P S}^{(\lambda)}$ and estimated covariance matrix of $\Psi^{(\lambda)}$ applied to Table 3a,b.

(a) For Table 3a

\begin{tabular}{cccccc}
\hline & \multicolumn{3}{c}{ Index } & \multicolumn{3}{c}{ Covariate Matrix } \\
\cline { 2 - 6 }$\lambda$ & $\hat{\boldsymbol{\Phi}}_{S}^{(\lambda)}$ & $\hat{\boldsymbol{\Phi}}_{\mathbf{P S}}^{(\lambda)}$ & $\hat{\sigma}_{\mathbf{1 1}}^{(\lambda)}$ & $\hat{\sigma}_{\mathbf{1 2}}^{(\lambda)}$ & $\hat{\sigma}_{\mathbf{2 2}}^{(\lambda)}$ \\
\hline-0.5 & 0.047 & 0.054 & 0.535 & 0.139 & 0.411 \\
0 & 0.077 & 0.089 & 1.305 & 0.315 & 1.029 \\
1 & 0.102 & 0.119 & 2.105 & 0.478 & 1.707 \\
\hline
\end{tabular}

(b) For Table 3b

\begin{tabular}{cccccc}
\hline & \multicolumn{2}{c}{ Index } & \multicolumn{3}{c}{ Covariate Matrix } \\
\cline { 2 - 6 }$\lambda$ & $\hat{\boldsymbol{\Phi}}_{S}^{(\lambda)}$ & $\hat{\boldsymbol{\Phi}}_{\boldsymbol{P S}}^{(\lambda)}$ & $\hat{\sigma}_{\mathbf{1 1}}^{(\lambda)}$ & $\hat{\sigma}_{\mathbf{1 2}}^{(\lambda)}$ & $\hat{\sigma}_{\mathbf{2 2}}^{(\lambda)}$ \\
\hline-0.5 & 0.009 & 0.036 & 0.040 & 0.010 & 0.110 \\
0 & 0.015 & 0.060 & 0.111 & 0.027 & 0.290 \\
1 & 0.020 & 0.082 & 0.208 & 0.048 & 0.513 \\
\hline
\end{tabular}

Figure 2 shows the confidence regions of $\Psi^{(\lambda)}$ applied to the data in Table 3a,b. We see that the confidence regions of $\Psi^{(\lambda)}$ do not overlap with regard to several values of $\lambda$. Therefore, it may be concluded that Table $3 a, b$ has a different structure with regard to the degree of deviation from DS, in the sense that Table $3 a, b$ has a different structure with regard to the degree of deviation from $\mathrm{S}$. However, we cannot conclude whether the degree of deviation from DS is greater for Table 3a than for Table $3 \mathrm{~b}$. This is because, when both the degrees of deviation from S and PS are greater for Table 3a than for Table 3b, we can conclude that the degree of deviation from DS is greater for Table 3a than for Table $3 b$. 


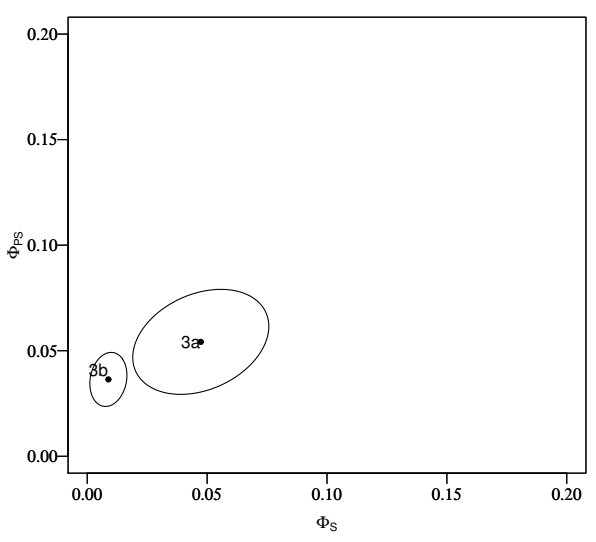

(a) $\lambda=-0.5$

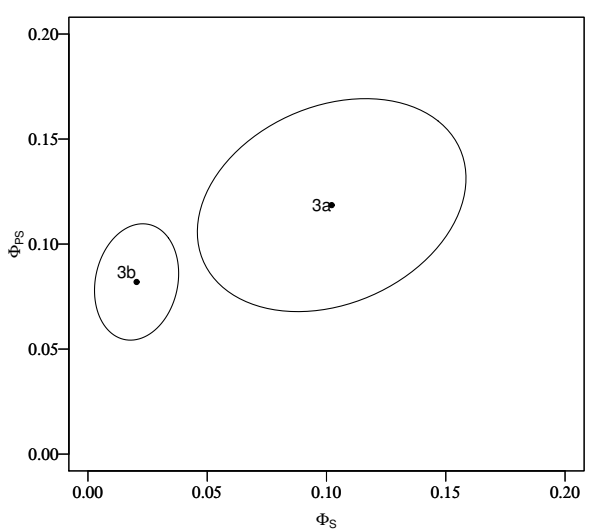

(c) $\lambda=1$

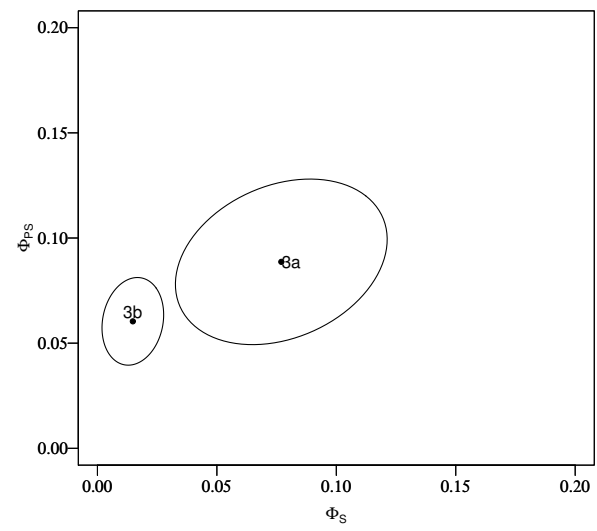

(b) $\lambda=0$

Figure 2. Approximate $95 \%$ confidence regions for $\Psi^{(\lambda)}$, applied to the data in Table 3a,b, where $\lambda=-0.5,0,1$.

Next, consider the data in Table 5, which are taken from Tomizawa et al. [15].

Table 5. The two tables below show the decayed teeth of 349 female 18-39-year-old patients of a dental clinic in Sapporo City, for the period 2001-2005; from Tomizawa et al. [15].

(a) For female with left and right decayed teeth

\begin{tabular}{ccccc}
\hline & \multicolumn{3}{c}{ Right } \\
\cline { 2 - 5 } Left & $\mathbf{0}$ to $\mathbf{4}$ & $\mathbf{5}$ to $\mathbf{8}$ & $\mathbf{9}$ and above & Total \\
\hline 0 to 4 & 103 & 45 & 1 & 149 \\
5 to 8 & 35 & 84 & 33 & 152 \\
9 and above & 3 & 17 & 42 & 62 \\
Total & 141 & 146 & 76 & 363
\end{tabular}

(b) For female with lower and upper decayed data

\begin{tabular}{ccccc}
\hline & \multicolumn{3}{c}{ Upper } \\
\cline { 2 - 5 } Lower & $\mathbf{0}$ to $\mathbf{4}$ & $\mathbf{5}$ to $\mathbf{8}$ & $\mathbf{9}$ and above & Total \\
\hline 0 to 4 & 97 & 62 & 15 & 174 \\
5 to 8 & 20 & 63 & 75 & 158 \\
9 and above & 2 & 6 & 23 & 31 \\
Total & 119 & 131 & 113 & 363 \\
\hline
\end{tabular}

We shall compare the degrees of deviation from DS for Table 5a,b using the confidence region for $\Psi^{(\lambda)}$. The estimates of $\Sigma^{(\lambda)}$, applied to the data in Table 5a,b, are shown in Table 6. 
Table 6. Estimated indexes $\hat{\Phi}_{S}^{(\lambda)}$ and $\hat{\Phi}_{P S}^{(\lambda)}$ and estimated covariance matrix of $\Psi^{(\lambda)}$ applied to Table 5a,b.

\begin{tabular}{cccccc}
\hline (a) For Table 5a & \multicolumn{3}{c}{ Covariate Matrix } \\
\cline { 2 - 6 }$\lambda$ & $\hat{\mathbf{\Phi}}_{S}^{(\lambda)}$ & $\hat{\boldsymbol{\Phi}}_{P S}^{(\lambda)}$ & $\hat{\sigma}_{\mathbf{1 1}}^{(\lambda)}$ & $\hat{\sigma}_{\mathbf{1 2}}^{(\lambda)}$ & $\hat{\sigma}_{\mathbf{2 2}}^{(\lambda)}$ \\
\hline-0.5 & 0.024 & 0.064 & 0.113 & 0.043 & 0.142 \\
0 & 0.040 & 0.105 & 0.302 & 0.127 & 0.361 \\
1 & 0.055 & 0.141 & 0.540 & 0.231 & 0.605 \\
\hline
\end{tabular}

(b) For Table 5b

\begin{tabular}{cccccc}
\hline & \multicolumn{2}{c}{ Index } & \multicolumn{3}{c}{ Covariate Matrix } \\
\cline { 2 - 6 }$\lambda$ & $\hat{\boldsymbol{\Phi}}_{S}^{(\lambda)}$ & $\hat{\boldsymbol{\Phi}}_{\mathbf{P S}}^{(\lambda)}$ & $\hat{\sigma}_{\mathbf{1 1}}^{(\lambda)}$ & $\hat{\sigma}_{\mathbf{1 2}}^{(\lambda)}$ & $\hat{\sigma}_{\mathbf{2 2}}^{(\lambda)}$ \\
\hline-0.5 & 0.281 & 0.233 & 0.962 & 0.541 & 0.472 \\
0 & 0.414 & 0.356 & 1.526 & 0.890 & 0.884 \\
1 & 0.501 & 0.445 & 1.718 & 1.067 & 1.127 \\
\hline
\end{tabular}

Figure 3 shows the confidence regions of $\Psi^{(\lambda)}$ applied to the data in Table $5 \mathrm{a}, \mathrm{b}$. We see that the confidence regions of $\Psi^{(\lambda)}$ do not overlap in both horizontal and vertical axes with regard to several values of $\lambda$. Therefore, we can conclude that the degree of deviation from DS is greater for Table $5 b$ than for Table $5 a$.

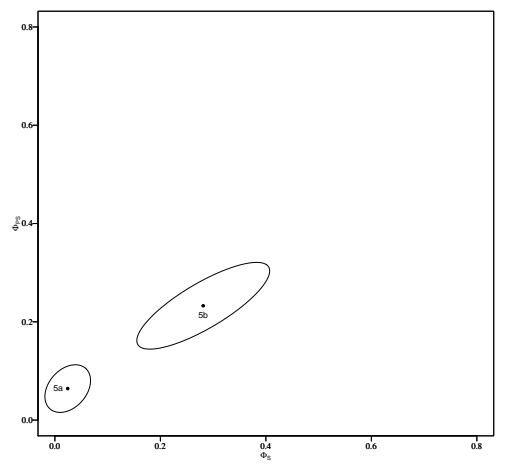

(a) $\lambda=-0.5$

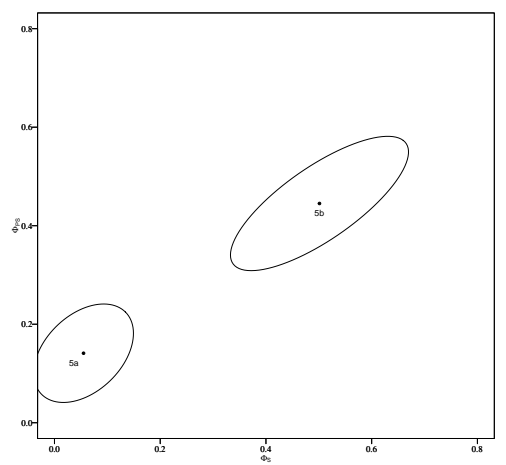

(c) $\lambda=1$

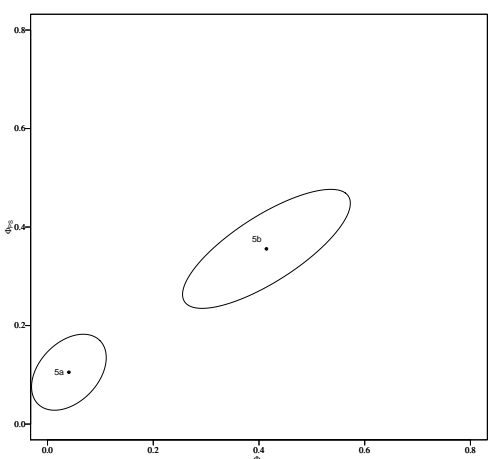

(b) $\lambda=0$

Figure 3. Approximate $95 \%$ confidence regions for $\Psi^{(\lambda)}$, applied to the data in Table $5 \mathrm{a}, \mathrm{b}$, where $\lambda=-0.5,0,1$.

\section{Concluding Remarks}

This study proposed a generalized two-dimensional index that concurrently measures the degree of deviation from S and PS. Since the two indexes $\left(\Phi_{S}^{(\lambda)}\right.$ and $\left.\Phi_{P S}^{(\lambda)}\right)$ were used to measure the degree of deviation from $S$ and PS are not independent $\left(\sigma_{12}^{(\lambda)} \neq 0\right)$, it 
is necessary to concurrently measure the degree of deviation from S and PS when we measure the degree of deviation from DS. To compare degrees of deviation from DS in several datasets using the proposed two-dimensional index, we should use several $\lambda$ rather than one specified $\lambda$. Therefore, we recommend to choose the several $\lambda$ (e.g., $-0.5,0,1$ ) corresponding to the famous divergence.

The estimator of the proposed two-dimensional index is the unbiased estimator when the sample size is large. When the sample size is small, however, the estimator of the proposed two-dimensional index may be the biased estimator. Through simulation study, Tomizawa et al. [16] investigated the performance of the estimator $\hat{\Phi}_{S}^{(\lambda)}$. Tomizawa et al. [16] showed that (1) when the sample size was less than 300, the estimator $\hat{\Phi}_{S}^{(\lambda)}$ had a bias, (2) when the sample size was above 300, it had a slight bias, and (3) when the sample size was above 1000, it had almost no bias. We believe that the proposed two-dimensional estimator $\hat{\mathbf{\Psi}}^{(\lambda)}$ may be similar results to the estimator $\hat{\Phi}_{S}^{(\lambda)}$, although it is necessary to verify by simulation study. In future research, the above concern will be investigated.

Author Contributions: Conceptualization, S.A. and S.T.; methodology, S.A. and H.H.; software, S.A. and H.H.; validation, A.I.; formal analysis, S.A. and H.H.; writing—original draft preparation, S.A. and H.H.; writing-review and editing, A.I. and S.T. All authors have read and agreed to the published version of the manuscript.

Funding: This research received no external funding.

Institutional Review Board Statement: Not applicable.

Informed Consent Statement: Not applicable.

Data Availability Statement: Not applicable.

Acknowledgments: The authors would like to thank anonymous reviewers and the editor for their comments and suggestions to improve this paper.

Conflicts of Interest: The authors declare no conflict of interest.

\section{Appendix A. Existing Index $\Phi_{S}^{(\lambda)}$}

Assuming that $\pi_{i j}+\pi_{j i}>0$ for all $i \neq j$, the index $\Phi_{S}^{(\lambda)}$, which represents the degree of deviation from $S$, is expressed as follows:

$$
\Phi_{S}^{(\lambda)}=\frac{\lambda(\lambda+1)}{2^{\lambda}-1} I_{S}^{(\lambda)}
$$

where

with

$$
I_{S}^{(\lambda)}=\frac{1}{\lambda(\lambda+1)} \sum_{i \neq j} a_{i j}\left[\left(\frac{a_{i j}}{b_{i j}}\right)^{\lambda}-1\right]
$$

$$
\delta=\sum_{i \neq j} \pi_{i j}, \quad a_{i j}=\frac{\pi_{i j}}{\delta}, \quad b_{i j}=\frac{\pi_{i j}+\pi_{j i}}{2 \delta}
$$

Appendix B. Existing Index $\Phi_{P S}^{(\lambda)}$

Assuming that $\pi_{i j}+\pi_{i^{*} j^{*}}>0$ for all $(i, j) \in E$, the index $\Phi_{P S}^{(\lambda)}$, which represents the degree of deviation from PS, is expressed as follows:

$$
\Phi_{P S}^{(\lambda)}=\frac{\lambda(\lambda+1)}{2^{\lambda}-1} I_{P S}^{(\lambda)}
$$


where

$$
I_{P S}^{(\lambda)}=\frac{1}{\lambda(\lambda+1)} \sum_{(i, j) \in E} \sum_{i j}\left[\left(\frac{c_{i j}}{d_{i j}}\right)^{\lambda}-1\right],
$$

with

$$
\Delta=\sum_{(i, j) \in E} \pi_{i j}, \quad c_{i j}=\frac{\pi_{i j}}{\Delta}, \quad d_{i j}=\frac{\pi_{i j}+\pi_{i^{*} j^{*}}}{2 \Delta} .
$$

Note that $I_{S}^{(\lambda)}$ and $I_{P S}^{(\lambda)}$ are the power divergence between the two conditional distributions, and the value at $\lambda=0$ is taken to be the limit as $\lambda \rightarrow 0$.

\section{References}

1. Bowker, A.H. A test for symmetry in contingency tables. J. Am. Stat. Assoc. 1948, 43, 572-574. [CrossRef] [PubMed]

2. Bishop, Y.M.M.; Fienberg, S.E.; Holland, P.W. Discrete Multivariate Analysis: Theory and Practice; The MIT Press: Cambridge, MA, USA, 1975.

3. Tan, T.K. Doubly Classified Model with R; Springer: Singapore, 2017.

4. Agresti, A. An Introduction to Categorical Data Analysis, 3rd ed.; Wiley: Hoboken, NJ, USA, 2018.

5. Wall, K.D.; Linert, G.A. A test for point-symmetry in J-dimensional contingency-cubes. Biom. J. 1976, 18, $259-264$.

6. Tomizawa, S. Double symmetry model and its decomposition in a square contingency table. J. Jpn. Stat. Soc. 1985, 15, 17-23.

7. Tomizawa, S.; Seo, T.; Yamamoto, H. Power-divergence-type measure of departure from symmetry for square contingency tables that have nominal categories. J. Appl. Stat. 1998, 25, 387-398. [CrossRef]

8. Tomizawa, S.; Yamamoto, K.; Tahata, K. An entropy measure of departure from point-symmetry for two-way contingency tables. Symmetry Cult. Sci. 2007, 18, 279-297.

9. Yamamoto, K.; Komatsu, M.; Tomozawa, S. Measure of departure from double-symmetry for square contingency tables. J. Stat. Appl. 2010, 5, 105-118.

10. Ando, S.; Tahata, K.; Tomizawa, S. A bivariate index vector for measuring departure from double symmetry in square contingency tables. Adv. Data Anal. Classif. 2019, 9, 519-529. [CrossRef]

11. Cressie, N.A.C.; Read, T.R.C. Multinomial goodness-of-fit tests, J. R. Stat. Ser. B 1984, 46, 440-464. [CrossRef]

12. Read, T.R.C.; Cressie, N.A.C. Goodness-of-Fit Statistics for Discrete Multivariate Data; Springer: New York, NY, USA, 1988.

13. Agresti, A. Categorical Data Analysis, 3rd ed.; Wiley: Hoboken, NJ, USA, 2013.

14. Andersen, E.B. Introduction to the Statistical Analysis of Categorical Data; Springer: Berlin, Germany, 1997.

15. Tomizawa, S.; Miyamoto, N.; Iwamoto, M. Linear column-parameter symmetry model for square contingency tables: Application to decayed teeth data. Biom. Lett. 2006, 43, 91-98.

16. Tomizawa, S.; Miyamoto, N.; Ohba, N. Improved approximate unbiased estimators of measures of asymmetry for square contingency tables. Adv. Appl. Stat. 2007, 7, 47-63. 